\title{
Risk Factors Associated with Prognosis in Patients with Acute Stroke: A Retrospective Observational Study
}

\author{
Fardin Faraji ${ }^{1,2,3}$, Pegah Mohaghegh ${ }^{4,}{ }^{*}$, Hosein Rafiee Ravandi ${ }^{5}$ and Javad Javaheri ${ }^{4}$ \\ ${ }^{1}$ Associate Professor of Neurology, Department of Neurology, School of Medicine, Arak University of Medical Sciences, Arak, Iran \\ 2 Traditional and Complementary Medicine Research Center, Arak University of Medical Sciences, Arak, Iran \\ ${ }^{3}$ Applied Neuroscience Research Center, Islamic Azad University, Arak Branch, Arak, Iran \\ ${ }^{4}$ Assistant Professor of Community and Preventive Medicine, Department of Community Medicine, School of Medicine, Arak University of Medical Sciences, Arak, Iran \\ ${ }^{5}$ Student in General Medicine, Students Research Committee, Arak University of Medical Sciences, Arak, Iran
}

* Corresponding author: Pegah Mohaghegh, Department of Community Medicine, School of Medicine, Arak University of Medical Sciences, Arak, Iran. Tel: 09120253653; Email: pmohaghegh@arakmu.ac.ir

Received 2020 October 24; Revised 2020 November 05; Accepted 2020 November 11.

\begin{abstract}
Background: Stroke is one of the major causes of disability and mortality in Iran. Therefore, it is of critical importance to know the factors affecting the effective management of acute stroke for better prevention and treatment of stroke.

Objectives: The present study aims at investigating the risk factors associated with prognosis in patients with acute stroke who were registered in the "724" stroke management registry system at Amir Al-Momenin Hospital, Arak, Iran.

Methods: This retrospective observational study included all acute stroke patients who were registered in the "724" stroke management registry system at Amir Al-Momenin Hospital, Arak, Iran, from April 2017 to April 2019. Data were analyzed in SPSS software (version 23) through the Chi-square test, Fisher's exact test, Mann Whitney U test, and logistic regression.

Results: This study investigated 139 patients with acute stroke registered in the "724" stroke management registry system at Amir AlMomenin Hospital, Arak, Iran, from April 2017 to April 2019. The mean age of the patients was $62.91 \pm 13.31$ years, and the majo rity of the cases were male ( $\mathrm{n}=77 ; 55.4 \%)$. Totally, $104(75.4 \%)$ and 31 (22.3\%) patients had hypertension and diabetes, respectively. In-hospital mortality showed a statistically significant relationship with a history of stroke, brain computed tomography scan results and high National Institutes of Health Stroke Scale (NIHSS) at baseline $(\mathrm{P}<0.05)$.

Conclusion: High rate of hypertension among stroke patients highlights its significance as an important modifiable risk factor for stroke. Attention to these risk factors is essential for the primary prevention of stroke. History of stroke and NIHSS score at baseline $\geq 8$ associated with poor outcome at discharge.
\end{abstract}

Keywords: IV-tPA, Prognosis, Risk factors, Stroke, Thrombolysis

\section{Background}

Stroke is one of the major causes of disability and the second major cause of mortality in Iran and throughout the world $(1,2)$. About two-thirds of stroke cases occur in developing countries. The annual prevalence rates of stroke in Iran are 139 and 144 cases per 100.000 population in males and females, respectively. Many important risk factors of stroke, such as hypertension, diabetes, hyperlipidemia, and smoking are modifiable and preventable (1-3). It is noteworthy that the age of stroke is about a decade earlier in Iran, compared to that in other countries; moreover, the annual rate of stroke is increasing in this country $(4,5)$. While the majority of strokes could be prevented or treated early, unfortunately, in third world countries, patients refer to the hospital when a disability has occurred from a stroke. This imposes significant tangible and intangible costs on the patient and the health system.

In 1996, the International Food and Drug Administration approved the use of venous thrombolytic therapy for the treatment of acute ischemic stroke. In the meantime, there were few reports on thrombolytic therapy in Asian and developing countries, including Iran (6). Lack of resources and infrastructures for thrombolytic injection in most hospitals and the late referral of patients are among the obstacles to thrombolytic injection in these countries, where only $30 \%$ of the patients are qualified for this injection (7). In Iran (2016), the Ministry of Health and Medical Education developed the standard protocol of treatment services for acute stroke (8).

According to this protocol, Alteplase was approved as the preferred thrombolytic medication for the treatment of acute ischemic stroke. In addition, the qualified candidate hospitals for stroke were obliged to provide the infrastructure required to inject the medication in the Stroke Care Unit to the patients who have referred within the golden time of less than $4.5 \mathrm{~h}$ and met the injection indications.

In this process, the acute stroke-suspected patient is referred to a qualified hospital, followed by the activation of the caring code "724". After examination and emergency brain computed tomography (CT) scan, IV-tPA (tissue plasminogen activator) injection will be administered to the ischemic stroke patients who met the indication criteria (8). Since 2017, Amir Al-Momenin Hospital in Arak, Iran, has been one of 
the qualified hospitals for the treatment of acute stroke patients and embraced the protocol for qualified patients.

\section{Objectives}

The present study aims at investigating the characteristics and clinical factors affecting outcomes of patients with acute stroke who were registered in the "724" stroke management registry system at Amir Al-Momenin Hospital, Arak, Iran.

\section{Methods}

This retrospective observational study included all acute stroke patients who were referred to Amir Al-Momenin Hospital in Arak, Iran, from April 2017 to April 2019 and registered in the "724" stroke management registry system. All patients diagnosed with acute stroke during this period were selected using the census method. Gender, age, place of residence, mode of referral to the hospital, time from onset of symptoms to hospital admission, underlying diseases, hospital services, risk factors, Modified Ranking Scale (MRS) and National Institutes of Health Stroke Scale (NIHSS) on arrival, type of stroke, history of using anticoagulants, IV-tPA receiving, and injection side-effects were assessed based on the information recorded in the "724" stroke management registry system.

Data were analyzed in SPSS software (version 23) through the Chi-square test, Fisher's exact test, Mann Whitney $U$ test, and logistic regression. Moreover, quantitative and qualitative variables were described by mean $\pm \mathrm{SD}$, as well as frequency and percentage, respectively.

\section{Results}

In total, 139 patients with acute stroke were registered in the "724" stroke management registry system at Amir Al-Momenin Hospital, Arak, Iran, from April 2017 to April 2019. The mean age of the patients was 62.91 \pm 13.31 years (age range: 25-91), and the majority of the cases $(n=77 ; 55.4 \%)$ were male. Moreover, the selected patients were refereed from Arak $(n=108 ; 77.7 \%)$ and other towns of the province $(\mathrm{n}=31 ; 22.3 \%)$ (Table 1$)$.

About $75.4 \%, 22.3 \%, 10.1 \%$, and $6.5 \%$ of the patients had hypertension, diabetes, hyperlipidemia, and a history of stroke respectively. Regarding the smoking status, $12.9 \%$ of the patients were smokers and $4.3 \%$ of the cases abused drugs. Considering the comparison of the risk factors and medical history between genders, smoking $(\mathrm{P}<0.001)$ and drug abuse $(\mathrm{P}=0.025)$ were higher in males, whereas females obtained higher levels of atrial fibrillation ( $\mathrm{P}=0.027)$. Additionally, the majority of the patients (55.4\%) were transported through emergency medical services. Out of 118 patients with ischemic stroke, 69 $(49.6 \%)$ cases were qualified for IV-tPA injection, and others denied to receive IV-tPA due to unwillingness, time delay in referral, old age, other diagnoses (transient ischemic attack [TIA] and hemorrhage), use of anticoagulants due to underlying diseases, coagulopathy, and neurologist's decision.

According to the results of the study (Table 2), the patient outcome (in-hospital mortality) correlated statistically with the history of stroke $(\mathrm{P}=0.002)$, NIHSS score at baseline $(\mathrm{P}=0.002)$, brain CT scan results $(\mathrm{P}=0.011)$, and hospitalization duration in a stroke unit $(\mathrm{P}=0.049)$. Moreover, there was a borderline statistically significant association

\begin{tabular}{|c|c|c|c|c|c|}
\hline & & \multicolumn{2}{|c|}{ Gender } & \multirow{2}{*}{ Total (\%) } & \multirow{2}{*}{ P-value } \\
\hline & & Male (\%) & Female (\%) & & \\
\hline \multirow[b]{2}{*}{ Age } & $25-49$ & $17(22.1)$ & $13(21.0)$ & $30(21.6)$ & \multirow{2}{*}{0.874} \\
\hline & $50-91$ & $60(77.9)$ & $49(79.0)$ & $109(78.4)$ & \\
\hline \multicolumn{6}{|c|}{ Known risk factors and comorbidities } \\
\hline Hypertension & Yes & $59(77.6)$ & $45(72.6)$ & $104(75.4)$ & 0.493 \\
\hline Smoking & Yes & $17(22.1)$ & $1(1.6)$ & $18(12.9)$ & $<0.001$ \\
\hline Diabetes & Yes & $14(18.2)$ & $17(27.4)$ & $31(22.3)$ & 0.193 \\
\hline Atrial Fibrillation & Yes & $3(3.9)$ & $9(14.5)$ & $12(8.6)$ & 0.027 \\
\hline Dyslipidemia & Yes & $7(9.1)$ & $7(11.3)$ & $14(10.1)$ & 0.668 \\
\hline Drug abuse & Yes & $6(7.8)$ & 0 & $6(4.3)$ & 0.025 \\
\hline Stroke history & Yes & $3(3.9)$ & $6(9.7)$ & $9(6.5)$ & 0.169 \\
\hline \multirow{3}{*}{$\begin{array}{l}\text { Anticoagulants used } \\
\text { prior to event }\end{array}$} & Warfarin & $3(3.9)$ & $7(11.3)$ & $10(7.2)$ & \multirow{3}{*}{0.198} \\
\hline & Heparin & $2(2.6)$ & $1(1.6)$ & $3(2.2)$ & \\
\hline & NOVAC & $5(6.5)$ & $1(1.6)$ & $6(4.3)$ & \\
\hline \multirow{4}{*}{$\begin{array}{l}\text { Mode of arrival to } \\
\text { emergency room }\end{array}$} & Ambulance & $41(53.2)$ & $36(58.1)$ & $77(55.4)$ & \multirow{4}{*}{0634} \\
\hline & Private car & $19(24.7)$ & $13(21)$ & $32(23)$ & \\
\hline & Transfer from other hospital & $17(22.1)$ & $12(19.4)$ & $29(20.23)$ & \\
\hline & In hospital stroke & $0(0)$ & $1(1.6)$ & $1(0.7)$ & \\
\hline \multirow{3}{*}{ Time delay Mean \pm SD } & Onset of symptoms to calling EMS (min) & $61.86 \pm 58.11$ & $51.45 \pm 34.89$ & $57.28 \pm 48.62$ & 0.956 \\
\hline & EMS to door (min) & $53.42 \pm 26.86$ & $45.91 \pm 19.79$ & $49.82 \pm 23.88$ & 0.302 \\
\hline & Onset of symptoms to door & $144.46 \pm 86.57$ & $121.73 \pm 70.13$ & $135.72 \pm 80.46$ & 0.544 \\
\hline $\begin{array}{l}\text { Thrombolysis } \\
\text { With IV-tPA }\end{array}$ & 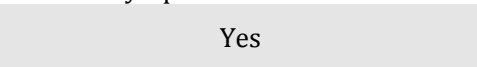 & $40(51.9)$ & $29(46.8)$ & $69(49.6)$ & 0.544 \\
\hline
\end{tabular}


between the outcome and MRS score at arrival $(\mathrm{P}=0.053)$ (Table 2). Out of 69 patients who received the thrombolytic injection, unintended side-effects of IV-tPA (intracerebral hemorrhage) were reported in five cases. Accordingly, a statistically significant correlation was found between the outcome and side effects of IV-tPA injection $(\mathrm{P}=0.000)$. On the other hand, the outcome had no statistically significant relationship with age, gender, and mode of transportation to the hospital $(\mathrm{P}>0.05)$.

In the multivariate logistic regression model (Forward Stepwise LR method), three variables, namely predictive NIHSS at baseline, stroke history, and hospitalization time in the stroke unit, helped predict the outcomes and remained in the model.
According to the model output in Table 3, the value of the odds ratio for the NIHSS 1 is $0 R=1.17$. This indicates that the patient's odds of death due to stroke increases by about 1.17 times for every 1point increase in the NIHSS score at baseline.

According to the model output, the value of the odds ratio for stroke history is $\mathrm{OR}=15.23$, which means that the patients with a history of the previous stroke will probably die 15.23 times more, compared to a person that has no history of stroke.

The next variable is the hospitalization duration in the stroke unit. The value of the odds ratio for this variable is obtained at $\mathrm{OR}=1.25$, which means that for each day of hospitalization of a stroke patient in the stroke unit, the odds of death increases by 1 .

\begin{tabular}{|c|c|c|c|c|}
\hline & \multicolumn{2}{|c|}{ Outcome } & \multirow[b]{2}{*}{ Total } & \multirow[b]{2}{*}{ P-value } \\
\hline & $\begin{array}{c}\text { Discharge } \\
\text { No. }(\%)\end{array}$ & $\begin{array}{c}\text { In-hospital mortality } \\
\text { No. (\%) }\end{array}$ & & \\
\hline \multicolumn{5}{|l|}{ NIHSS at arrival } \\
\hline $0-8$ & $17(13.2)$ & $1(10)$ & $18(12.9)$ & \multirow{3}{*}{0.002} \\
\hline $8-16$ & $98(76)$ & $4(40)$ & $102(73.4)$ & \\
\hline$>16$ & $14(10.9)$ & $5(50)$ & $19(13.7)$ & \\
\hline \multicolumn{4}{|l|}{ MRS at arrival } & \multirow{3}{*}{0.053} \\
\hline $4>($ good $)$ & $90(69.8)$ & $4(40)$ & $94(67.6)$ & \\
\hline$\leq 4$ (weak) & $39(30.2)$ & $6(60)$ & $45(32.4)$ & \\
\hline \multicolumn{4}{|l|}{ Brain CT scan } & \multirow{6}{*}{0.011} \\
\hline Normal & $49(38)$ & 0 & $49(35.3)$ & \\
\hline Suspected & $45(34.9)$ & $5(50)$ & $50(36)$ & \\
\hline Ischemia under $1 / 3$ & $13(10.1)$ & 0 & $13(9.4)$ & \\
\hline Ischemia up to $1 / 3$ & $4(3.1)$ & $2(20)$ & $6(4.3)$ & \\
\hline Hemorrhage & $18(14)$ & $3(30)$ & $21(15.1)$ & \\
\hline \multicolumn{4}{|l|}{ Stroke type } & \multirow{3}{*}{0.172} \\
\hline Ischemic & $111(86)$ & $7(70)$ & $118(84.9)$ & \\
\hline Hemorrhagic & $18(14)$ & $3(30)$ & $21(15.1)$ & \\
\hline \multicolumn{4}{|l|}{ Thrombolysis with IV-tPA } & \multirow{3}{*}{0.197} \\
\hline No & $63(48.8)$ & $7(70)$ & $70(50.4)$ & \\
\hline Yes & $66(51.2)$ & $3(30)$ & $69(49.6)$ & \\
\hline \multicolumn{4}{|c|}{ Intracerebral hemorrhage after venous Thrombolysis } & \multirow{3}{*}{0.000} \\
\hline No & $63(95.5)$ & $1(33.3)$ & $64(92.8)$ & \\
\hline Yes & $3(4.5)$ & $2(66.7)$ & $5(7.2)$ & \\
\hline \multicolumn{3}{|l|}{ History of stroke } & & \multirow{3}{*}{0.002} \\
\hline No & $123(95.5)$ & $7(70)$ & $130(93.5)$ & \\
\hline Yes & $6(4.7)$ & $3(30)$ & $9(6.5)$ & \\
\hline $\begin{array}{l}\text { Length of hospitalization (day) } \\
\text { Mean } \pm \text { SD }\end{array}$ & $5.78 \pm 4.66$ & $9.20 \pm 11$ & $6,02 \pm 5.38$ & 0.341 \\
\hline Hospitalization in a stroke unit (day) & $2.09 \pm 2.86$ & $6.80 \pm 11.84$ & $2.42 \pm 4.27$ & 0.049 \\
\hline Emergency department (hour) & $3.36 \pm 9.69$ & $2.70 \pm 1.76$ & $3.31 \pm 9.35$ & 0.218 \\
\hline Door to needle time (min) & $62.53 \pm 38.60$ & $127.67 \pm 110.50$ & $65.63 \pm 44.81$ & 0.336 \\
\hline
\end{tabular}

Table 3. Multivariable logistic regression results regarding predictors of in-hospital mortality in patients with acute stroke

\begin{tabular}{|c|c|c|c|c|}
\hline \multirow{2}{*}{ Variables } & \multirow{2}{*}{$\mathbf{P}$} & \multirow{2}{*}{ OR } & \multicolumn{2}{|c|}{ 95\% C.I } \\
\hline & & & Lower & Upper \\
\hline NIHSS at baseline & 0.005 & 1.173 & 1.050 & 1.312 \\
\hline History of stroke & 0.005 & 15.235 & 2.318 & 100.132 \\
\hline Hospitalization duration in the stroke unit & 0.019 & 1.125 & 1.019 & 1.241 \\
\hline
\end{tabular}

\section{Discussion}

This study evaluated 139 patients with acute stroke. The mean age of the patients was $62.91 \pm 13.31$ years, and $21.6 \%$ of the cases were younger than 50 years old. Hypertension was the most prevalent risk factor that was observed in $75.4 \%$ of the patients. In total, $22.3 \%$ of the individuals had diabetes, and $12.9 \%$ of the cases were smokers. As observed, the most common risk factor for stroke was hypertension, which was consistent with the results of other similar studies $(2,9,10)$. In the present study, smoking and substance abuse were more prevalent in males; however, females obtained higher levels of 


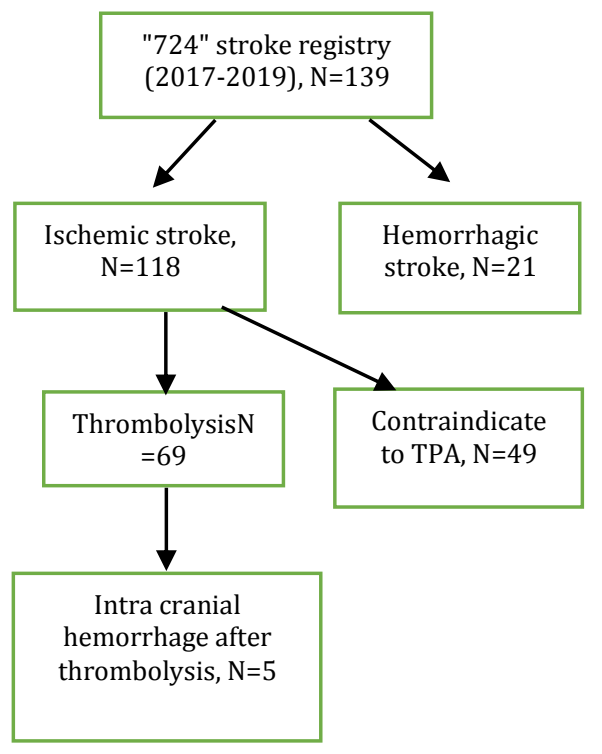

Figure 1. Study flowchart

atrial fibrillation. In a study conducted by Talebi et al., the risk factors of hypertension and diabetes were more prevalent in females; however, the majority of the males were smokers (10).

The mean hospital stay in the present study was $6.02 \pm 5.38$ days; however, in a study conducted by Fahoudi et al., it was reported to be 17.3 days (9). In this study, $84.9 \%$ and $15.1 \%$ of the patients had ischemic and hemorrhagic strokes, respectively, followed by a hospital mortality rate of 7.2\%.

According to the results of a study carried out by Daneshfard et al. in Namazi Hospital, Shiraz, Iran, $88.2 \%$ and $11.8 \%$ of the patients had ischemic and hemorrhagic strokes, respectively (1). Similarly, Farhoodi et al. in Tabriz reported that $76.5 \%$ of all patients had an ischemic stroke with an in-hospital mortality rate of $20.5 \%$ (9).

In the same vein, Ovisgaran et al. in Isfahan, Iran, indicated an in-hospital mortality rate of $16.5 \%$; moreover, the mortality rate after 28 days was estimated at $25.6 \%$ in this study. The results also revealed that the mortality rate was higher in females, the elderly, and patients with a history of stroke (11). According to the findings of the present study, the mortality rate was lower, compared to the results of the aforementioned studies.

The duration from the onset of symptoms to patient arrival at Amir Al-Momenin Hospital, Arak, Iran, was $135.72 \pm 80.48 \mathrm{~min}$, and $49.6 \%$ of all patients were qualified for IV-tPA injection. In a study performed by Ghiyasian et al. at Farshchian Hospital, Hamadan, Iran, the mean time of arrival at the hospital was $4.77 \mathrm{~h}(286.2 \mathrm{~min})$, and $36.2 \%$ of patients had been transported to the hospital within $2 \mathrm{~h}$ after stroke (12).

The results of a study conducted by Khorvash et al. in Isfahan, Iran, revealed that only $10.7 \%$ of the patients were referred to the hospital within $4.5 \mathrm{~h}$ after the onset of symptoms. Totally, $68.9 \%$ of these patients were qualified for IV-tPA; however, due to the failure to meet the golden time, the injection was performed only in $6.6 \%$ (13). According to the findings of other studies, the reasons for the late referral of the patients include pre-hospital emergency barriers, distance from the treatment center, nocturnal stroke, lack of information, and poor public knowledge of timely referral (12). Time is one of the major factors influencing the treatment of patients with acute stroke; moreover, the shortening of the time between the onset of symptoms and arrival at the hospital is effective in reducing the negative effects of stroke (14).

In the present study, there was a significant correlation between the NIHSS score at baseline and the outcome. Sadeghi Hokmabadi et al. investigated the factors affecting the prognosis of patients with stroke in Imam Reza Hospital, Tabriz, Iran. They found a significant correlation between the NIHSS score in patients with ischemic stroke and the outcome (15), which was consistent with the results of the present study.

Furthermore, a significant correlation was revealed between intracranial hemorrhage in IV-tPA recipients and outcomes in the present study. In the same line, Mehrpour et al. conducted a study on 37 patients who were treated with intravenous thrombolysis. In total, nine (24\%) patients showed hemorrhagic transformations in control brain CT after $24 \mathrm{~h}$ and seven $(18 \%)$ cases had intracerebral hemorrhages (ICH) without any deterioration of neurologic symptoms (asymptomatic ICH). Additionally, the outcomes of patients with symptomatic post-r-TPA hemorrhages were worse, compared to the nonhemorrhagic post-r-TPA patients (16). According to the studies, the use of thrombolytic medications in patients with acute ischemic stroke improves the prognosis and functioning of the patients. It also significantly increased the risk of ICH $(16,17)$.

The results of a meta-analysis conducted by Yoy $\mathrm{S}$ et al. in 2017 showed that the patients with mild ischemic stroke treated with venous thrombolytic therapy had a much better functional outcome based on the MRS; meanwhile, the risk of symptomatic intracranial hemorrhage was higher in these patients (OR=10.13); however, mortality rate showed no increase in this regard (OR=0.78) (17). The decision for the thrombolytic injection in the ischemic stroke patients depends on variables, such as patient's age, time of hospitalization, systolic blood pressure, diabetes, stroke intensity, comorbidities, and NIHSS (18). The early treatment of the thrombolytic after ischemic stroke (best time: 3-4.5 h) leads to the improvement of the positive effects of treatment and disability induced by the ischemic stroke (19). According to the literature, it is necessary to monitor the patient precisely from the injection to at least 48 
$\mathrm{h}$ after treatment to reduce the side effects of TPA injection (16-20).

Feng et.al (2015) conducted a multicenter study on the consequences of acute ischemic stroke treated with r-TPA. The multivariate logistic regression showed that an increase in the NIHSS score increased the risk of adverse outcomes by 8.75. This corresponding value was obtained at 1.17 in the current study (21). In a study titled "Predictors after Alteplase Thrombolysis Treatment in Acute Stroke Patients", Das et al. showed that a history of TIA increased the risk of death in the patients by 6 times. Similarly, the present study demonstrated that a history of stroke increased the risk of death by 15.2 times. On the other hand, Das et al. showed that for a 1-point increase in the NIHSS scale during accepting the patient, the risk of death increased by 8 times; however, in our study, this corresponding number was determined at 1.17 (22).

\subsection{Limitations}

One of the limitations of the present study was the absence of data on NIHSS and MRS scores of patients at discharge, as well as from patient arrival to hospital to the performance of brain CT scan. If the data on NIHSS and MRS scores are recorded after treatment, accurate decisions can be made on the patients' prognosis. Moreover, the number of patients was insufficient to judge the association of thrombolysis complications with the outcome (inhospital mortality) Therefore, it is recommended that future studies be conducted with a larger sample size.

\section{Conclusion}

The high rate of hypertension among patients with stroke highlights its significance as an important modifiable risk factor for stroke. Attention to these risk factors is essential for the primary prevention of stroke. A poor outcome at discharge was associated with a history of stroke and NIHSS score at baseline $\geq 8$.

\section{Acknowledgments}

This study was extracted from a dissertation by a general medical student with an ethic code of IR.ARAKMU.REC.1398.023. The authors are grateful to the Research Deputy, Arak University of Medical Sciences, Arak, Iran.

\section{Footnotes}

Authors' Contribution: Pegah Mohaghegh and Fardin Faraji were responsible for conception and design. Hosein Rafiee Ravandi and Fardin Faraji were responsible for data collection. Pegah Mohaghegh was responsible for writing the manuscript. Pegah Mohaghegh, Fardin Faraji, and Javad Javaheri were responsible for analysis and interpretation. Fardin Faraji was responsible for the critical revision of the paper. All authors were responsible for the final approval of the paper. Pegah Mohaghegh had the overall responsibility.

Conflict of Interests: The authors declare no conflict of interest in this study.

Ethical Approval: This study has been approved by the Ethics Committee of Arak University of Medical Sciences, Arak, Iran, with an ethic code of IR.ARAKMU.REC.1398.023.

\section{Funding/Support: Nil.}

Informed consent: In this study, we used data registered in the "724" stroke management registry system, which was without mentioning the name of patients. However, written informed consent was obtained from legally authorized representatives before the study.

\section{References}

1. Daneshfard B, Izadi S, Shariat A, Toudaji MA, Beyzavi Z, Niknam L. Epidemiology of stroke in Shiraz, Iran. Iran J Neurol. 2015;14(3):158-63. [PubMed: 26622981].

2. Ghiasian M, Daneshyar S, Lotfi yar M. Assessment of one-year prognosis in patients with stroke. J Mazandaran Uni Med Sci. 2019;28 (168):172-6.

3. World Health Organization. Bulletin of the World Health Organization. Geneva. World Health Organization; 2020.

4. Dehghani Firoozabadi M, Kazemi T, Sharifzadeh G, Dadbeh S, Dehghan P. Stroke in Birjand, Iran: a hospital-based study of acute stroke. Iran Red Crescent Med J. 2013;15(3):264-8. doi: 10.5812/ircmj.4282. [PubMed: 23984011].

5. Ghandehari K. Epidemiology of Stroke in Iran. Galen Med J. 2016;5(S1):3-9.

6. Baratloo A, Forouzanfar M M, Hashemi B, Safari S, Delavar Kasmaei $\mathrm{H}$, et al. Tissue plasminogen activator: a literature review. Arch Neurosci. 2016;3(1):e30452. doi: 10.5812/archne urosci.30452

7. Shahjouei S, Bavarsad-Shahripour R, Assarzadegan F, Rikhtegar R, Mehrpour M, Zamani B, et al. Acute management of stroke in Iran: Obstacles and solutions. Iran J Neurol. 2017;16(2):62-71. [PubMed: 28761627].

8. Ministry of health and medical education . "Standard service manual for the treatment of acute Stroke".Curative affair of Ministry of health and medical education. First edition. Published by Kelkekhial. Tehran.2016.

9. Farhoudi M, Mehrvar K, Sadeghi-Bazargani H, Hashemilar M, Seyedi-Vafaee M, Sadeghi-Hokmabad E, et al. Stroke subtypes, risk factors and mortality rate in northwest of Iran. Iran J Neurol. 2017;16(3):112-7.

10. Talebi M, Ghertasi M, Taheraghdam A, Andalib S, Sharifipour E. A comparison of risk factors and severity of ischemic stroke in female and male genders in North-West Iran: A cross-sectional study. Iran J Neurol. 2014;13(4):215-9. [PubMed: 25632333].

11. Oveisgharan S, Ghaemmaghami AB, Bahonar A, Sarrafzadegan $\mathrm{N}$. Case fatality rate and disability of stroke in Isfahan, Iran: Isfahan stroke registry. Iran J Neurol. 2016;15(1):9-15. [PubMed: 27141271].

12. Ghiasian M, Mazaheri S, Hashemi Rahbarian F. Factors delaying hospital arrival aftr acutestroke onset. Avicenna J Clin Med. 2017;23(4):293-9. doi: 23043-hums/21859.1.

13. Khorvash F, Heidary F, Saadatnia M, Chitsaz A, Tolou-Ghamari Z. Tissue plasminogen activator; identifying major barriers related to intravenous injection in ischemic acute cerebral infraction. J Res Med Sci. 2017;22(1):19. doi: 10.4103/17351995.200318. [PubMed: 28458710].

14. Nagendra CV, Kumar TS, Bohra V, Wilben V, Karan V, Huded V. 
Factors affecting the effective management of acute stroke: a prospective observational study. Indian J Crit Care Med. 2018;22(3):138-43. doi: 10.4103/ijccm.IJCCM_232_17. [PubMed: 29657369].

15. Sadeghi-Hokmabadi E, Yazdchi M, Farhoudi M, Sadeghi H, Taheraghdam A, Rikhtegar R, et al. Prognostic factors in patients with acute ischemic stroke treated with intravenous tissue plasminogen activator: The first study among Iranian patients. Iran J Neurol. 2018;17(1):31-7. [PubMed: 30186557].

16. Mehrpour M, Aghaei M, Motamed MR. Safety and feasibility of intravenous thrombolytic therapy in Iranian patients with acute ischemic stroke. Med J Islam Repub Iran. 2013; 27(3):113-8. [PubMed: 24791120].

17. You S, Saxena A, Wang X, Tan W, Han Q, Cao Y, et al. Efficacy and safety of intravenous recombinant tissue plasminogen activator in mild ischaemic stroke: a meta-analysis. Stroke Vasc Neurol. 2018;3(1):22-7. doi: 10.1136/svn-2017-000106. [PubMed: 29600004].

18. Tolou Ghamari Z, Mazdak H. Pharmacotherapy Updates of Recombinant Tissue Plasminogen Activator (r-TPA) in Acute Ischemic Stroke. Jentashapir J Health Res. 2016;7(3):e36067.
19. Abbasi V, Atalu A, Sharghi A, Taghvatalab F. A clinical study investigation the three months prognosis of patients with ischemic stroke treated with recombinant tissue plasminogen activator (rt-PA) and its effective factors. J Emergen Pract Trauma. 2019;5:47-50. doi: 10.15171/jept.2019.05.

20. Tan G, Wang H, Chen S, Chen D, Zhu L, Xu D, et al. Efficacy and safety of low dose alteplase for intravenous thrombolysis in Asian stroke patients: a meta-analysis. Sci Rep. 2017; 7(1):16076. doi: 10.1038/s41598-017-16355-9. [PubMed: 29167555].

21. Ling-feng LA, Yun WA, Zheng BA, Bao-rong WA, Hui-rong LI. Clinical Outcomes of Intravenous rt-PA Thrombolysis Therapy for Advance-Aged Patients with Acute Ischemic Stroke: A Multi-Center Clinical Study.J Int Transl Med. 2015,3(3):200-3. doi: 10.11910/2227-6394.2015.03.03.09.

22. Das S, Mondal GP, Bhattacharya R, Ghosh KC, Das S, Pattem $\mathrm{HK}$, et al. Predictors of postthrombolysis outcome and symptomatic postthrombolysis hemorrhage following intravenous thrombolysis with alteplase for acute ischemic stroke. J Neurosci Rural Pract. 2020;11(2):315-24. doi: 10.1055/s-0040-1709946. [PubMed: 32367987]. 